\title{
Low radio frequency spectrum of Sgr-A*
}

\author{
Subhashis Roy \\ NCRA-TIFR, Pune University Campus, \\ Pune-411007, India \\ email: roy@ncra.tifr.res.in
}

\begin{abstract}
.
We observed the Galactic centre (GC) region with the partially upgraded Giant Metrewave Radio Telescope (GMRT) using a wideband system in frequency ranges of $300-500 \mathrm{MHz}$ with 16 antennas. Preliminary results are presented here. Sgr-A* is clearly detected down to $450 \mathrm{MHz}$. Sgr-A West slowly disappears at lower frequencies across the band. By taking cross-cuts across the known major-axis of Sgr- $\mathrm{A}^{*}$, we measure its total flux density across the band to be $0.4 \mathrm{Jy}$ consistent with what is expected from earlier results. It clearly indicates lack of absorption from Sgr-A West. Its spectral index is consistent with its higher frequency value of +0.3 .
\end{abstract}

Keywords. Galaxy: center - radio continuum: ISM - H II regions

\section{Introduction}

The Sgr-A complex is seen within a few arc-min of the centre of the Galaxy. From radio observations, it is known to be composed of three sources along our line of sight. They are, (i) a compact radio source, Sgr-A* seen coincident with the dynamical centre of the Galaxy. Around this object is the Sgr-A West HII region with a size of about a few arc-min. Around this HII region is the supernova remnant Sgr-A East. Sgr-A* is coincident with the central supermassive black hole and has been studied in all the observable bands. Sgr-A* was clearly detected at $620 \mathrm{MHz}$ (Roy \& Rao 2004), but its presence at $330 \mathrm{MHz}$ has been inferred from cross-cuts (Nord et al. 2004). The low radio frequency spectrum of Sgr-A* below $1 \mathrm{GHz}$ remains uncertain and its better determination will provide important information on its intrinsic optical depth as well as external environment through free-free absorption. The spectrum determined from a single wideband observation is free from source variability and absolute flux density calibration uncertainties at different frequencies carried out on different days. Here we present our preliminary study of the region with wide band observation from 300-500 $\mathrm{MHz}$ with the GMRT.

\section{Observations and data reduction}

Observations were carried out with the partially upgraded GMRT using the new wideband feeds between 300 to $500 \mathrm{MHz}$. Sixteen of the 30 antennas could be used. 3C48 was used as primary flux density calibrator, and 1714-252 was used to calibrate the antenna based complex gain variations in time and frequency. To keep the output power constant during observation, antenna based attenuators were used to lower the gain of each antennas by a specific amount while observing the GC region. Data was corrected for this known gain change. Data reduction was carried out in AIPS. Imaging was carried out by dividing the data in 6 equal parts across the band. 

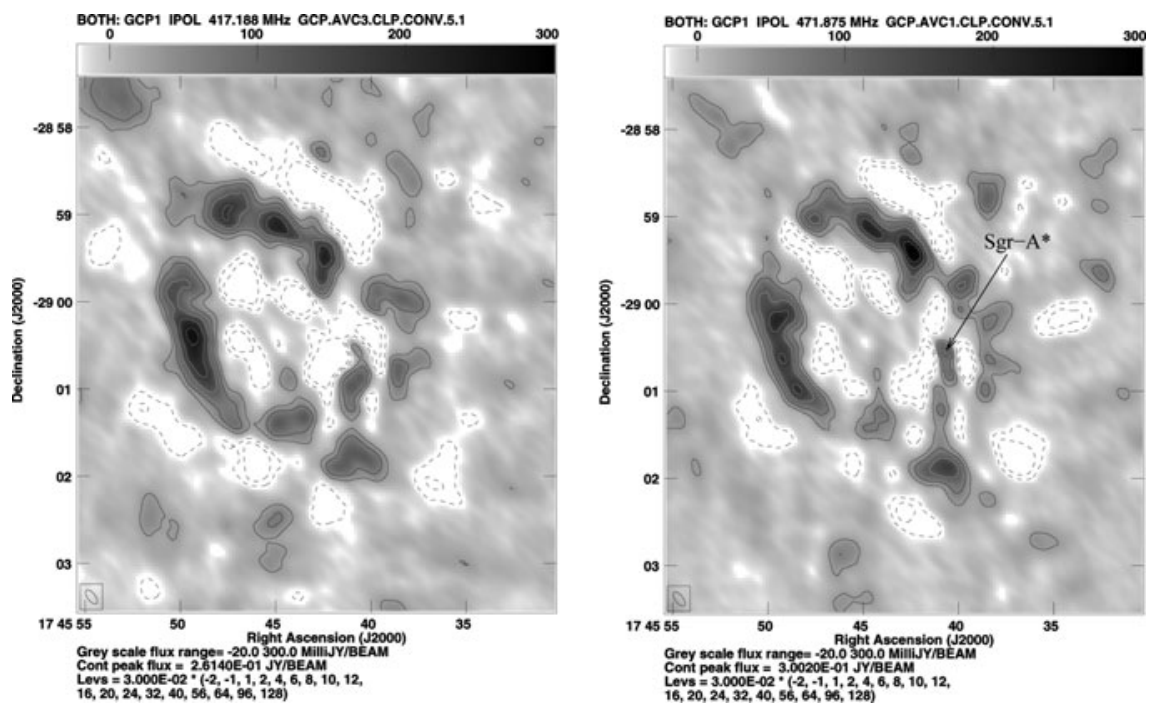

Figure 1. Images of Sgr-A complex at frequencies of 417 (left) and $472 \mathrm{MHz}$ (right). UV range of $1.5-28 \mathrm{k} \lambda$ was used to resolve out structures with angular size $>2^{\prime}$. Maps have a resolution of $11^{\prime \prime} .35 \times 5^{\prime \prime} .36$ with a position angle of $37.5 \mathrm{deg}$. Rms noises in the images are $\sim 5 \mathrm{mJy} / \mathrm{beam}$ (left) and $4 \mathrm{mJy} /$ beam (right).

\section{Results and Discussions}

Contour and gray scale map of Sgr-A complex at mid and high frequency part of the $300-500 \mathrm{MHz}$ band is shown in Fig. 1. Sgr-A* is clearly detected at a frequency of 472 $\mathrm{MHz}$. It could detected visually till about $450 \mathrm{MHz}$, which is significantly lower than earlier direct detection from its images at $620 \mathrm{MHz}$ (Roy \& Rao 2004).

\subsection{Flux density of $S g r-A^{*}$ at different frequencies}

Flux density of Sgr-A* at $472 \mathrm{MHz}$ is found to be consistent with what is expected from other frequencies $(0.4 \mathrm{Jy})$. To check for its presence in the presence of large scale absorption features from Sgr-A West at lower frequencies, following Nord et al. (2004), we took cross-cuts across Sgr-A* along its known major axis. These cross-cuts show that as the frequency decreases, the peak flux density of Sgr-A* falls by a large fraction. Despite its visual non-detection below about $450 \mathrm{MHz}$, peaks in cross cuts at lower frequencies could be identified at its expected position. Its total flux density is estimated from its peak value corrected after removal of a baselines and its expected major and minor axis size extrapolated from higher frequency Observations (Lo et al. 1998) by assuming its scattering size $\propto \lambda^{(11 / 5)}$. Measured flux densities at 472, 444, 417, 390, 363 and 335 $\mathrm{MHz}$ are $0.4 \mathrm{Jy}$ in all these bands. The measured flux density at $335 \mathrm{MHz}$ is consistent with Nord et al. (2004). No significant absorption by Sgr-A* at these frequencies are indicated by these measurements. A slightly inverted spectral index of +0.3 determined from higher frequencies is consistent with our measurements.

\section{References}

Nord, M. E., Lazio, T. J. W., Kassim, N. E., et al. 2004, AJ, 128, 1646

Roy, S. \& Pramesh Rao, A. 2004, MNRAS, 349, L25

Lo K. Y., Shen Z., Zhao J., \& Ho P. T. P., 1998, ApJL, 508, L61 